\title{
CIÊNCIA DA INFORMAÇÃO E DESIGN DE INTERAÇÃO: AS INTERLOCUÇÕES DAS DUAS ÁREAS EM PROJETOS DE BIBLIOTECAS DIGITAIS.
}

\author{
Karine Coutinho Drumond \\ Mestranda da Ciência da Informação na Universidade \\ Federal de Minas Gerais (UFMG). Designer, especialista \\ em Design de Interação. \\ karinedrumond@gmail.com \\ https://orcid.org/0000-0002-7645-5357
}

\author{
Célia da Consolação Dias \\ Professora Adjunta do Departamento de Organização e \\ Tratamento da Informação e Coordenadora do \\ Programa de Pós-Graduação em Gestão \\ \& Organização do Conhecimento (PPG-GOC) na \\ Escola de Ciência da Informação da Universidade \\ Federal de Minas Gerais (UFMG) \\ celiadias@gmail.com \\ https://orcid.org/0000-0003-0891-6454
}

\begin{abstract}
RESUMO
A resolução de problemas complexos da atual sociedade da informação exige uma abordagem multidisciplinar e uma atitude de colaboração entre as áreas do conhecimento. $O$ projeto de biblioteca digital é um destes problemas complexos, uma vez que combina aspectos tecnológicos, estruturais, de organização da informação e, também, de design. Partindo do pressuposto de interlocução entre a Ciência da Informação e do Design de Interação, este estudo visa responder às seguintes questões: "Quais interlocuções e elos existem entre as duas áreas?" e "Quais contribuições esta interlocução apresenta para projetos de bibliotecas digitais?" A partir de uma pesquisa bibliográfica, foram levantados possíveis elos e, posteriormente, criou-se um mapa conceitual, a fim de descrever, qualitativamente, e identificar as relações e interlocuções que se estabelecem entre as duas áreas, em projetos de bibliotecas digitais. Conclui-se que a interlocução acontece em duas dimensões principais: (1) dimensão histórica, uma vez que as áreas compartilham das influências histórias que as originaram e (2) dimensão de projeto. O projeto de biblioteca digital envolve conhecimentos do ponto de vista de organização e representação da informação, questões centrais da $\mathrm{Cl}$, quando a necessidade de compreender as necessidades de usuários para projetar melhores interfaces e interações entre sistemas e pessoas (questão central do design). Outros elos, também são discutidos, como os estudos com usuários, a interação homem-computador, a cognição, a usabilidade e design centrado no usuário.
\end{abstract}

Palavras-chave: Ciência da Informação. Design de Interação. Bibliotecas Digitais. Interação. Usuário da informação.

\section{INFORMATION SCIENCE AND INTERACTION DESIGN: THE INTERLOCUTIONS OF THE TWO AREAS IN DIGITAL LIBRARY PROJECTS}

\begin{abstract}
The resolution of complex problems in today's information society requires a multidisciplinary approach and an attitude of collaboration between areas of knowledge. The digital library project is one of these complex problems, since it combines technological, structural, information organization and also design aspects. Based on the assumption of interlocution between Information Science and Interaction Design, this study aims to answer the following question: "What interlocutions and links are there between the two areas?" And "What contributions does this interlocution present for digital library projects? A bibliographic research was conducted and possible links were raised and a conceptual map was subsequently created in order to qualitatively describe and identify the relationships and interlocutions that are established between the two areas in digital library projects. The results show that interlocution are two dimensions: (1) historical dimension, since the areas share the historical influences that originated them and (2) project dimension: the digital library project involves knowledge both from the point of view of organization and information representation, central issues of Cl. Also they share the need to understand the user's needs to design better interfaces and interactions between systems and people (central design issue). Other links are also discussed, such as user's studies, human-computer interaction, usability and user-centered design.
\end{abstract}


Keywords: Information Science. Interaction Design. Digital Libraries. Interaction. Information user.

\section{INTRODUÇÃO}

Sabe-se que o projeto de bibliotecas e repositórios digitais envolve muitos conhecimentos e desafios complexos. Os desafios vão desde decisões tecnológicas sobre plataforma, arquitetura de software, interoperabilidade, a questões envolvendo as necessidades dos usuários e a usabilidade dos sistemas, dentre outras. (CHOWDHURY, 2003). Atuar em cenário dinâmico, mutante e complexo, como os de hoje, exige uma abordagem múltipla, revelando a necessidade de interação constante da Ciência da Informação com outras áreas do conhecimento científico.

De um lado, a evolução tecnológica dos sistemas de recuperação da informação e das bibliotecas digitais permitiu maior disponibilidade de informações. De outro, no contexto digital, ela também apresenta desafios, como questões sobre o design das interfaces, a facilidade de uso dos sistemas e qualidade das interações entre usuários e tecnologia, em diversos dispositivos. Muitas pesquisas são realizadas, tanto na Ciência da Informação, quanto em outras áreas do conhecimento, com intuito de melhorar a experiência e satisfação dos usuários de bibliotecas digitais. Neste artigo, propomos um olhar para as possíveis interfaces de duas áreas do conhecimento: a Ciência da Informação e o Design de Interação, no contexto dos projetos de bibliotecas digitais. A escolha das duas áreas e a motivação para este estudo ocorreu pela experiência de uma das autoras que é Designer de Interação. A autora pesquisa o design de sistemas de busca e recuperação da informação, na perspectiva da Ciência da Informação, na pós-graduação de Gestão e Organização do Conhecimento, na Universidade Federal de Minas Gerais.

A Ciência da Informação $(\mathrm{Cl})$, pela sua possibilidade de interlocução com outras disciplinas, apresenta oportunidades para resolução de problemas complexos na sociedade de informação. Para Saracevic (1999), a Ciência da Informação tem características interdisciplinares, uma forte relação com a tecnologia da informação e dimensão social e humana. A área de Interação Homem-Computador (IHC) e a disciplina de Design de Interação (DI), por sua vez, propõem processos de design para criar sistemas interativos mais adequados aos usuários. (PREECE et al., 2005). 
A partir do pressuposto da existência de conexão entre a Ciência da Informação e o Design de Interação, as questões principais investigadas, neste estudo, são: "Quais as interlocuções e elos existem entre as duas áreas?" e "Quais as contribuições esta interlocução pode apresentar para projetos de bibliotecas digitais?".

\section{METODOLOGIA}

O objetivo deste estudo é analisar as interlocuções entre a Ciência da Informação e - Design de Interação em projetos de bibliotecas digitais. Para investigar as questões propostas neste estudo, foi realizada uma pesquisa bibliográfica com vistas abuscar 0 aporte teórico para subsidiar as análises. A partir desta pesquisa, foi traçado um breve histórico das duas áreas e elucidados, conceitualmente, seus elos e relações em projetos de bibliotecas digitais. Os resultados desta investigação, bem como os elos encontrados, são apresentados de duas formas:textualmente, no corpo do texto, e também, por meio de um mapa conceitual.

O mapa conceitual é uma técnica de organização do conhecimento. Segundo Lima (2004), ele pode ser entendido como uma representação gráfica visual:

que descreve a relação das ideias do pensamento, relação esta pré-adquirida ao longo do processo de aprendizagem na construção do conhecimento. (LIMA, 2004, p. 135).

Os mapas conceituais podem ser utilizados para diversas finalidades, como por exemplo: para gerar ideias, para desenhar uma estrutura complexa de maneira mais amigável, facilitando a estrutura de documentos, para estruturar e comunicar ideias na forma gráfica, entre outras funções. (LIMA, 2004). Neste artigo, o mapa conceitual foi utilizado, portanto, no final do processo de pesquisa bibliográfica, como forma de representar, visualmente, a síntese dos conceitos identificados, a partir da questão de pesquisa.

Com base nas questões propostas, foram identificados os principais conceitos-chave e teorias que subsidiaram o percurso metodológico desta investigação. A primeira parte apresenta um breve histórico da $\mathrm{Cl}$, das bibliotecas e dos repositórios digitais e, na sequência da temática, os estudos de usuários. Em seguida,discute-se sobre o Design de Interação e os elementos envolvidos no design para bibliotecas digitais. Por fim, são discutidos os resultados da análise e apresentados os pontos de contato entre as duas áreas. 
O processo de criação do mapa conceitual seguiu as etapas propostas em Novak (2008), citados por Lima (2004): (1) seleção, que inclui a escolha do assunto e identificação das palavras-chave; (2) ordenação: quando se faz a organização de conceitos, do mais abstrato para o mais concreto; (3) agrupamento: quando se reúne os conceitos em um mesmo nível de abstração e com forte interelacionamento; (4) arranjo: neste faz-se a organização de conceitos na forma de um diagrama; (5) link e preposição: trata-se da conexão de conceitos, com linhas e nomeação de cada linha, com uma proposição.

\section{AS BIBLIOTECAS DIGITAIS E A CIÊNCIA DA INFORMAÇÃO}

Segundo Saracevic (1999), a história recente das bibliotecas e repositórios digitais está ligada a própria história da $\mathrm{Cl}$ como área do conhecimento. $\mathrm{O}$ surgimento da $\mathrm{Cl}$, como área formal de estudo científico, inicia-se na revolução técnico-científica, que ocorre após a Segunda Guerra Mundial, em meados do século XX. Este mesmo autor cita eventos históricos, que marcaram o surgimento da área, tais como o artigo de Vannevar Bush, cientista do Massachusetts Institute of Technology (MIT), que, em 1945, tratou do problema, ainda hoje crucial para a $\mathrm{Cl}$ : a explosão informacional e a tarefa de tornar acessível o acervo, cada vez maior, de conhecimento. Também propôs uma máquina chamada MEMEX, uma solução tecnológica que teria a capacidade de "associar ideias" e de replicar o processo mental, artificialmente. O conceito, inclusive, influenciou, mais tarde, o surgimento dos sistemas de hipertexto e a própria inteligência artificial.

Os Estados Unidos tiveram papel central no desenvolvimento da $\mathrm{Cl}$, uma vez que o Congresso americano e as agências governamentais financiaram e desenvolveram programas e reuniram esforços em larga escala, nos anos de 1950 e 1960. Os programas tinham como intuito investigar soluções para resolver os problemas gerados pela explosão informacional. Tais esforços foram coordenados sob a ótica da importância estratégica da informação para o governo e a sociedade.

As áreas financiadas pelas iniciativas do governo americano incluíam estudos e avaliação de soluções de Recuperação de Informação (RI) nos anos de 1950 e 1960. A partir dos anos 1990, se intensificam as iniciativas de criação das bibliotecas digitais e, a partir de então, a RI passa a ser tema central para a Cl. 
As soluções técnicas desenvolvidas aos longos dos anos variaram enormemente: dos cartões perfurados aos $C D-R O M s$, dos sistemas não interativos aos sistemas web com múltiplas possibilidades de interação e a inteligência artificial. Observou-se que a evolução que se seguiu também ocorreu nos formatos "[...] de bases documentais para bases de conhecimento, dos textos escritos aos multimídias, da recuperação de citações à recuperação de textos completos e ainda aos sistemas inteligentes de perguntas e respostas". (SARACEVIC, 1999. p. 44).

O surgimento das Novas Tecnologias da Informação e Comunicação (NTICs) provoca, então, mudanças na forma de produção, transmissão, recuperação e uso da informação. As tecnologias que mais provocam mudanças nesse contexto são a Internet e os sistemas de hipertextos. (MARQUES, 1995). As novas tecnologias e a própria Web permitem que acervos, antes confinados em espaços físicos, pudessem estar acessíveis e disponibilizados, em larga escala.

Chowdhury (2003) observa que as definições do que são as bibliotecas digitais se encaixam em duas perspectivas principais: a) as que enfatizam o armazenamento e o uso de conteúdos digitais e b) as definições que enfocam na abordagem das bibliotecas digitais, como serviço de informação. Borgman (1999), por exemplo, aponta que pesquisadores, advindos das áreas de Engenharia e Ciências da Computação, definem as bibliotecas digitais, como o processo tecnológico de coletar e recuperar conteúdos em nome da comunidade de usuários. Enquanto que os bibliotecários tendem a compreender as bibliotecas, enquanto instituições ou serviços. Borgman (1999, p. 234) define as bibliotecas digitais como:

uma extensão e aprimoramento de sistemas de armazenamento e recuperação de informações que manipulam dados digitais em qualquer meio (vídeo, texto, som, imagem estática ou dinâmica) e existem em redes distribuídas.

Arms (2000, p. 2) destaca a noção gerencial de acervo e serviços. Assim, para o autor, "uma biblioteca digital é uma coleção gerenciável de informação, com serviços associados, onde as informações são armazenadas em formatos digitais e acessível pela rede." A visão que integra as duas perspectivas (serviço e tecnologia) é dada por Gladney (1994), nos seguintes termos:

[...] é uma mistura de computação digital, armazenamento e maquinário de comunicação, que juntamente com conteúdo e software necessário para reproduzir, emular e estender os serviços promovidos pelas bibliotecas 
convencionais baseadas em papel e outras formas de coletar, catalogar, buscar e disseminar informação. Uma biblioteca digital completa precisa oferecer todos os serviços essenciais e explorar as vantagens do armazenamento, sistema de busca e comunicação digital. (GLADNEY, 1994, p. 2).

As bibliotecas digitais podem envolver, também, recursos de informação em formatos impressos, como livros e outros registros, além de formatos digitais. Nesse sentido, elas podem ser também conhecidas como bibliotecas híbridas. (CHOWDHURY, 2003).

Marchionini (1995) acrescenta que o funcionamento de uma biblioteca digital ocorre em um contexto complexo de projeto, moldado por quatro dimensões: comunicação, tecnologia, serviços e conteúdo. O serviço seria o ponto central, e, segundo o autor, poderia ser aprimorado, ao fornecer melhores serviços de referência, respostas em tempo real, ajuda sob demanda e outros mecanismos de envolvimento maior, com usuários. Breivik e Gee (1989) propõem que as bibliotecas digitais devem ser pensadas como espaços que favoreçam a construção de novas aprendizagens e conhecimentos, nas quais o uso da informação visa impulsionar a capacidade das pessoas de resolverem problemas e transformarem a própria realidade.

De maneira geral, pode-se dizer que a problemática da explosão da informação, inserida no contexto maior da evolução da sociedade da informação, a partir dos estudos realizados, contribuiu para o crescimento e a expansão das fronteiras da $\mathrm{Cl}$. Ela passa a compartilhar seus problemas, cada vez mais complexos, também com outras áreas. O campo dos estudos com usuários, dentro da $\mathrm{Cl}$, é um exemplo de um destes campos de estudos, que fazem interlocução e colaboração com outras áreas.

\subsection{Os estudos de usuários na Ciência da Informação}

A necessidade de compreender os usuários da informação já está presente na Ciência da Informação, mesmo antes da revolução técnico-científica, que se seguiu nos anos de 1950 e 1960. Esses estudos já aconteciam na arquivologia, museologia e biblioteconomia - campos interligados historicamente à Ciência da Informação. (SIRIHAL, 2012). A diferença é que, nos anos 1950 e 1960, a Cl focou nos trabalhos relacionados à recuperação da informação e em fenômenos básicos da informação. Nos anos de 1970, o paradigma deslocou-se para uma direção mais ampla, envolvendo estudos de usuários e suas interações. (SARACEVIC, 1999). Essa abordagem inclui estudos 
sobre os usuários, usos, situações, contextos e interações das pessoas com as informações, para além do foco somente na tecnologia dos sistemas de recuperação.

Essa linha e comunidade que se formou ficou conhecida como "centrada no humano" (user-centered ou human-centered, no inglês). A outra abordagem ficou conhecida como "centrado no sistema" (System-centered). Esta, por sua vez, focava mais nos aspectos dos sistemas computacionais. Esses aspectos são notados por Saracevic (1999) como uma falta de interação entre os dois campos, dentro da $\mathrm{Cl}$ :

A questão é como entregar e incorporar o design que melhor atenda aos usuários, integrando às funcionalidades do sistema e usar as vantagens proporcionadas por ambos, humanos e tecnologia?. (SARACEVIC, 1999, p. 1057).

Estudos mais recentes, como os de Gasque e Costa (2010), sugerem uma mudança relevante na terminologia adotada na área, que passou de "estudos de usuários" ou "necessidades e uso de informação" para "comportamento informacional de usuários". Os autores destacam que "trata-se, contudo, não somente de alteração terminológica, mas, sobretudo, de mudança paradigmática, resultado de transformações no modo como o tópico é definido e abordado e na forma como é investigado". (Ibidem, p.21).

Os autores identificam as mudanças significativas no foco dos trabalhos, culminando nos seguintes pontos: a) pesquisas mais centradas no indivíduo; b) inclusão de outros grupos estudados, além de cientistas e tecnólogos; c) abordagem multifacetada, englobando os aspectos sociocognitivos e organizacional; d) compreensão do comportamento informacional, como processo em que os indivíduos estão, constantemente, buscando e usando informações; e) ampliação dos estudos qualitativos, assim como do uso de múltiplos métodos; f) maior consistência teórica, com aumento de fundamentação interdisciplinar, g) crescimento do número de pesquisas, em todas as partes do mundo.

A partir desta breve perspectiva evolutiva dos estudos com usuários, é possível perceber a evolução na direção de uma abordagem mais multidimensional. Neste sentido, o comportamento informacional é "compreendido como processo natural do ser humano no papel de aprendiz da própria vida, requer visão ampla do pesquisador." (GASQUE E COSTA, 2010, p. 22). O que pode ser entendido como a necessidade de entender a interação, em determinado espaço-tempo,como um processo dinâmico. Os indivíduos se engajam nessas ações, quando têm necessidade de informação. Quando a interação ou mediação 
ocorre por meio de sistemas computacionais, a área de Interação Homem-Computador (IHC) e o campo mais recente do Design de Interação possuem contribuições.

\section{A INTERAÇÃO HOMEM-COMPUTADOR E O DESIGN DE INTERAÇÃO}

O IHC é um campo de estudo que se desenvolveu nos anos de 1980 e "que se preocupa com o design, avaliação e implementação de sistemas computacionais interativos para uso humano e com estudo de fenômenos importantes que o rodeiam". (ACM SIGCHI, 1992, p. 6). A história do IHC está, por sua vez, ligada à da Usabilidade, como pode ser percebido na linha do tempo, elaborada e adaptada, a partir de Dumas, (2007) e Grudin (2011), apresentada no Quadro 1.

Quadro 1 - Linha do Tempo IHC e Usabilidade

1911 Frederick Taylor publica Principles of Scientific Management, descreve estudos e métodos sobre tempo e movimento com foco em melhorar a eficiência industrial.

1943 Alphonse Chapanis, um tenente do Exército dos Estados Unidos, mostra que o "erro do piloto" pode ser bastante reduzido, por meio do layout mais intuitivo dos cockpits dos aviões.

${ }_{1957}$ A Sociedade de Fatores Humanos e Ergonomia é fundada e é considerada a maior associação científica do mundo para profissionais de fatores humanos / ergonomia.

Os laboratórios de empresas, como a IBM, realizam o que hoje chamamos de teste de 1979 usabilidade. A primeira publicação científica com usabilidade em seu título aparece, "O impacto comercial da usabilidade em sistemas interativos", de John Bennett.

1983 A primeira Conferência de IHC foi realizada em Boston, como parte do subgrupo "Special Interest Group on Computer-Human Interaction" (SIGCHI) da ACM.

John Whiteside, da Digital Equipment Corporation, e John Bennett, da IBM, publicaram uma 1988 série de capítulos e artigos sobre o tema "engenharia de usabilidade", que enfatizaram a definição antecipada de objetivos, prototipagem e avaliação iterativa.

1991 A Usability Professionals Association (UPA) é formada.

Começam a surgir trabalhos focando na experiência dos usuários. Os livros Observing the 2000 User Experience de Mike Kuniavsky e "Measuring theUser Experience" de Tom Tullis and Bill Albert são publicados.

2012 A UPA muda seu nome para "User Experience Professionals Association" (UxPA). Fonte: elaborado e adaptada a partir de Dumas, (2007) e Grudin (2011).

Como uma disciplina formal, o Design de Interação tenta encontrar seu espaço e delimitação, dentre outras disciplinas consideradas irmãs, como a Arquitetura da Informação (Al), Design de Interfaces, Design industrial, Design Gráfico, Fatores Humanos, Usabilidade, entre outras. (SAFFER, 2010).

O Design de Interação propõe processos de design para criar sistemas interativos mais adequados aos usuários. (PREECE et al, 2005). A Interaction Design Association (IxDA) - Associação dos Designers de Interação - definiu em 2004, o design de interação 
como sendo "a atividade de definir a estrutura e o comportamento de produtos e serviços interativos e suas interações com as pessoas". Segundo definição do IxDA, "produto interativo", são os artefatos envolvendo tecnologia computacional e tecnologia da informação. De forma complementar, Preece et al (2005) entende o DI como o "design de produtos interativos que fornecem suporte às atividades cotidianas das pessoas, seja no lar ou no trabalho." Designers de interação projetam para a possibilidade de uma interação. A interação propriamente acontece entre pessoas, máquinas e sistemas, em uma variedade de combinações. (SAFFER, 2010).

Uma possível forma de interpretar a diferença entre o DI e o IHC é compreender o DI com uma ênfase maior no processo de design, no processo de concepção, enquanto o IHC tem, tradicionalmente, uma ênfase maior em estudos de avaliação e suas implicações para o design. Preece et al (2005, p. 33) enumera algumas atividades básicas envolvidas no processo de design de interação:

1. Identificar necessidades dos usuários e estabelecer requisitos;

2. Desenvolver soluções alternativas que preencham os requisitos;

3. Construir versões interativas das soluções, de maneira que possam ser comunicados e avaliados; e

4. Avaliar o que está sendo construído durante o processo de desenvolvimento do sistema.

Saffer (2010, p. 6-8) complementa e afirma que, além das atividades básicas do design de interação, apontadas por Preece et al (2005), há, ainda no processo de DI, as seguintes características:

1. Foco nos usuários: os designers sabem que os usuários finais são o verdadeiro foco das soluções;

2. Uso de protótipos e ideação: designers exploram soluções e comunicam suas ideias por meio de protótipos, rascunhos, esquemas e outras representações visuais. Estes recursos são usados não apenas para materializar ideias, mas também como ferramenta para melhor compreensão do problema, sendo solucionado; e

3. Colaboração: Designers, normalmente, colaboram com outros profissionais para materializar soluções. (SAFFER, 2010, p. 6-8). 
Fallman (2003) descreve a atividade do designer como um processo iterativo de diálogo entre o problema e a solução. Neste processo, a atividade de criação de esboços e de protótipos estão no cerne do papel do designer. O design de interação é, portanto, um processo iterativo, possui abordagem centrada nos usuários e preocupa-se com a qualidade da interação e experiência dos usuários com sistemas e artefatos interativos.

\section{MAPA CONCEITUAL: OS ELOS ENTRE A CIÊNCIA DA INFORMAÇÃO E O DESIGN DE INTERAÇÃO}

Este estudo partiu do pressuposto de conexão entre os dois campos, $\mathrm{Cl}$ e DI e procurou identificar as possíveis interlocuções e elos existentes entre as duas áreas. A Figura 1 apresenta, em um mapa conceitual, as relações, os elos e as interlocuções identificadas, a partir da pesquisa bibliográfica realizada.

O primeiro elo identificado é caracterizado de uma perspectiva histórica. A Cl e o DI compartilham do mesmo panorama histórico, que impulsionou as duas áreas. As duas áreas do conhecimento foram influenciadas pela revolução técnico-científica, que ocorre após a Segunda Guerra Mundial, em meados do século XX. Desenvolve-se, neste período, a Ciência da Informação, Ciência da computação, Ciência Cognitiva, Interação-HumanaComputador (IHC), entre outras. (DUMAS, 2007; GRUDIN, 2011; SARACEVIC, 1999).

Além deste elo histórico, há, em comum, o foco nos usuários, refletido nos estudos de usuários e nos estudos sobre processos cognitivos. (ALBERGARIA et al, 2013). Por intermédio do desenvolvimento da tecnologia da informação, cresce, a partir dos anos de 1970, os estudos com usuários em projetos de sistemas computadorizados, tanto na perspectiva da $\mathrm{Cl}$ quanto no $\mathrm{IHC}$, com envolvimento dos primeiros testes de usabilidade. Com este foco no ser humano, tanto a $\mathrm{Cl}$ quanto o DI passam a trabalhar em problemas comuns, para projetos envolvendo tecnologia e informação, como as bibliotecas digitais. (SIRIHAL, 2012; SARACEVIC, 1999; GASQUE E COSTA, 2010; GRUDIN, 2011; PREECE et al, 2005).

A interação dos usuários em bibliotecas digitais é outro elo importante entre $\mathrm{Cl}$ e DI. A interface dos sistemas de recuperação da informação é o principal ponto de contato entre Cl e DI. (GASQUE E COSTA, 2010; PREECE et al, 2005; MARCHIONINI, 1995). 
Figura 1 - Elos e interlocuções entre $\mathrm{Cl}$ e DI em projetos de bibliotecas digitais

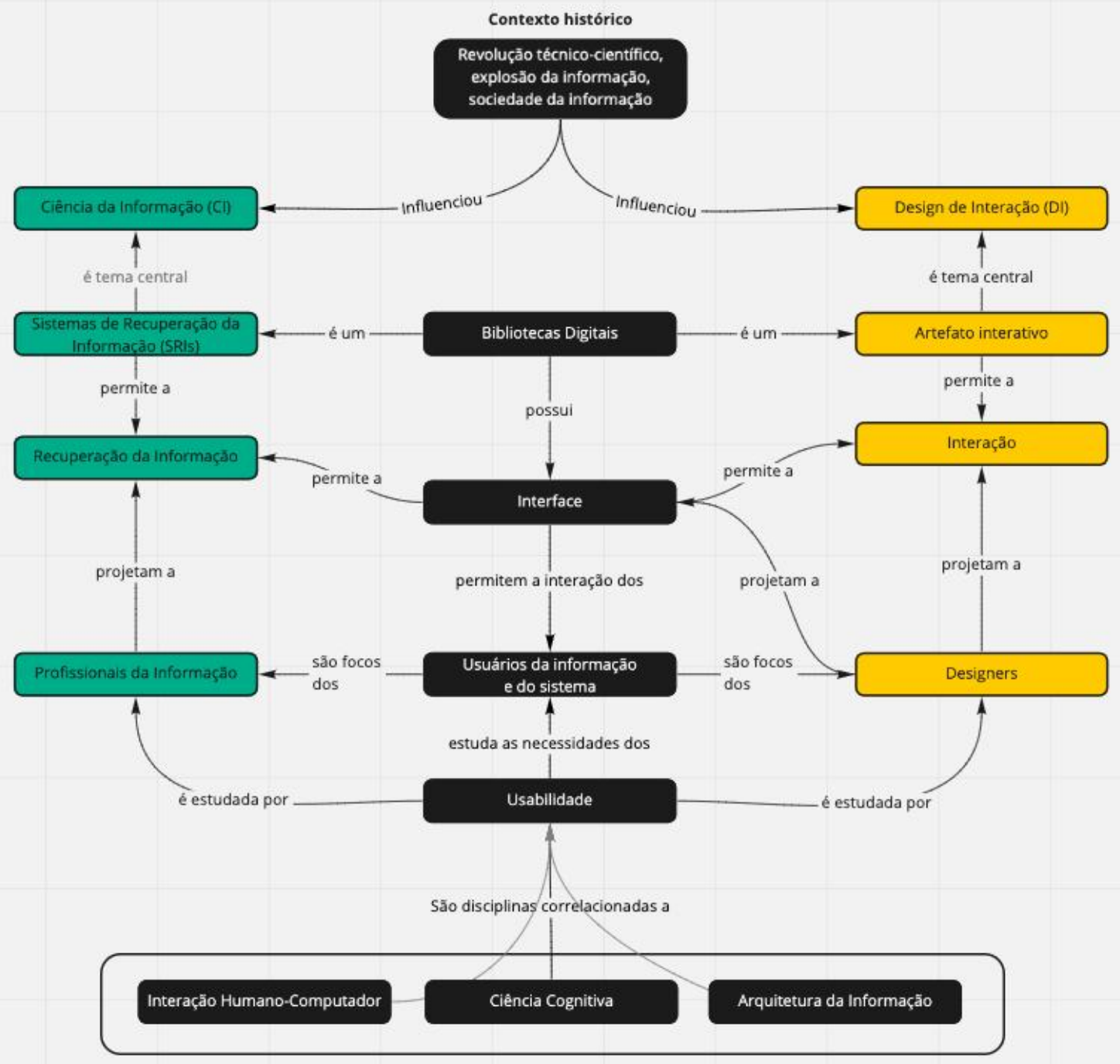

Fonte: Autora

Por fim, outro ponto de interlocução é a disciplina de usabilidade. Tal disciplina é objeto de estudos na Cl, na Ciência da Computação (CC) e também no Design. Tanto na Cl quanto na CC, a usabilidade está ligada às disciplinas de Interação Homem-Computador (IHC), desenvolvimento de sistemas de informação, centrados nos usuários e nos estudos sobre qualidade de software. No caso da $\mathrm{Cl}$, os aspectos da usabilidade estão presentes nos estudos de necessidades, comportamento de busca e uso de informação, além de avaliação de sistemas. (SARACEVIC, 2004; ALBERGARIA et al., 2013). No Design, a usabilidade aparece na literatura consultada, no Design de Interação e no Design de Interfaces, duas disciplinas correlacionadas. Em ambas, a usabilidade está presente no processo de criação e avaliação de sistemas interativos centrados nos usuários. (PREECE et al., 2005; NORMAN, 1986). 


\section{CONSIDERAÇÕES FINAIS}

Saracevic (1999) chamava atenção para a falta de comunicação e conexão entre as duas linhas (a centrada no sistema e a centrada nos usuários) e em projetos de bibliotecas digitais. Ou seja, a questão-chave, apontada pelo autor, pergunta como poderíamos fazer a abordagem centrada no usuário, unificada com a abordagem de desenvolvimento de tecnologias de informação. As práticas de design e a interação Homem-computador se fortalecem e evoluem, justamente, a partir desta problemática e da necessidade de se desenvolver soluções tecnológicas, centradas nas necessidades humanas, com melhor usabilidade e experiência de uso.

Popper (1989) afirmava que "nós não somos estudantes de um algum assunto nós somos estudantes de problemas e qualquer problema pode atravessar as fronteiras de qualquer assunto ou disciplina". Compartilhando dessa ideia, percebemos que $\mathrm{Cl}$ e DI comungam dos mesmos problemas complexos. Atuar em projetos complexos, como os sistemas de bibliotecas digitais,exige que os profissionais que atuam nesses projetos atravessem as fronteiras da disciplina. Com isso, pode-se afirmar que, compreendendo o que envolve o design e o projeto de bibliotecas digitais, percebe-se como as duas áreas podem se complementar e interagir.

A Cl contribui com conhecimentos para soluções teóricas e metodológicas de organização, representação, arquitetura, recuperação da informação e modelos teóricos sobre o comportamento informacional dos usuários, dentre outros. Por outro lado, o DI pode oferecer conhecimentos e técnicas para concepção, prototipação de soluções, aliados aos conhecimentos das áreas relacionadas à usabilidade e à interação homem-computador. O design, centrado no usuário, como abordagem de projeto, pode oferecer o elo entre os estudos com usuários e a eficiência das soluções de tecnologia para projetos de bibliotecas digitais.

É possível perceber, a partir do estudo, os inúmeros elementos comuns nas duas disciplinas e como se complementam em alguns aspectos, no projeto de bibliotecas digitais. A interlocução acontece em projetos e, também, nos estudos acadêmicos, quando compartilham dos temas, envolvendo sistemas da informação, interfaces de busca, acesso à informação e usabilidade.

Em relação à metodologia utilizada no mapa conceitual, é importante frisar que a sua construção é uma atividade iterativa. A evolução do mapa conceitual criado necessita 
de ciclos constantes de revisões e de ajustes, à medida que avançam os conhecimentos e as novas informações podem ser agregadas. Também foi confirmada, pelas autoras, a relevância do uso do mapa conceitual para a finalidade pretendida no artigo. Ou seja, o mapa conceitual, foi útil para representar, visualmente, a síntese dos elos identificados, a partir da questão de pesquisa e auxiliou a consolidar o conhecimento desenvolvido.

Este estudo abordou os elos e pontos de interlocução entre as duas áreas, a partir da análise histórica, das problemáticas estudadas e das contribuições das áreas em projetos de bibliotecas digitais. No entanto, não se pretendia desenvolver um estudo aprofundado destas variáveis. Novos estudos podem ser realizados no sentido de explorar a interlocução entre as áreas, com base na união entre teoria e prática, com estudos de caso, por exemplo. Também é desejável a evolução e o aprimoramento do mapa conceitual criado, de acordo com novos estudos futuros. Portanto, espera-se que o panorama traçado e os elos identificados, neste artigo, possam orientar estudos futuros e incentivar a colaboração das áreas em projetos diversos.

Atuar em cenário dinâmico, fluido, mutante e complexo, como os de hoje, exige uma abordagem dinâmica e em colaboração com outras áreas de conhecimento. Mais do que nunca percebemos a necessidade de parcerias e dinamismo entre as duas áreas do conhecimento.

\section{REFERÊNCIAS}

ARMS, W. Digital Libraries. Cambridge, MA: MIT Press, 2000.

BORGMAN, C. L. What are digital libraries? Competing visions. v. 4573, n. 98, 1999.

BREIVIK, P. S.; GEE, E. G. Information literacy: revolution in the library. New York: Collier Macmillan, 1989.

BUSH, V. As we may think. Atlantic Monthly, 176(1), 1945. P. 101-108.

CHOWDHURY, G. G; CHOWDHURY, S. Introduction to digital libraries. $2^{\circ}$. ed. London: Publishing, Facet, 2003.

CUNHA, M. B; AMARAL, S. A; DANTAS, E. B. Manual de estudos de usuários da informação. São Paulo: Atlas, 2015.

DUMAS, J. The great leap forward: The birth of the usability profession (1988-1993). Journal of Usability Studies; 2(2), 2007. P. 54-60

FALLMAN, D. Design-oriented human-computer interaction. Proceedings of the conference on Human factors in computing systems - CHI '03, n. February, p. 225, 2003. Disponível em: <http://portal.acm.org/citation.cfm?doid=642611.642652>. Acesso em: 8 Fev. 2020.

GASQUE, K. C.; COSTA, S. M. Evolução teórico-metodológica dos estudos de comportamento informacional de usuários. Ciência da Informação, v. 39, n. 1, jan./abr. 2010. P. 21-32. 
GLADNEY, H. M. et al. Digital library: gross structure and requirements: report from a March 1994 Workshop, San Antonio, mar, 1994. Disponível em:

<http://www.csdl.tamu.edu/DL94/paper/fox.html>. Acesso em: 20 nov. 2019.

GRUDIN, J. A Moving Target: The Evolution of HCI. In: Jacko, J., Ed., The Human-Computer Interaction Handbook, 3rd Edition, Taylor \& Francis, New York, 2011.

LIMA, G.A. B. Mapa Conceitual como ferramenta para organização do conhecimento em sistema de hipertextos e seus aspectos cognitivos. Perspectivas em Ciência da Informação, v. 9, 2004. P. 134-145.

MARCHIONINI, G. Information seeking in eletronic environments. Cambrigde: University Press, 1995.

MARQUES, E. V. Introdução aos sistemas de hipertexto. Revista da Escola de Biblioteconomia da UFMG, Belo Horizonte, v. 24, n. 1, jan./jun, 1995. P. 85-111

NOVAK, J. D.; CAÑAS, A. J. The Theory Underlying Concept Maps and How to Construct Them. Florida Institute for Human and Machine Cognition, 2008, 36 p. Disponível em:<http://cmap.inmc.us/Publications/ResearchPapers/TheoryUnderlyingConceptMaps.pdf>. Acesso em: 1 maio. 2020.

POPPER, K.R. Conjectures and refutations (5th ed.). London: Rutledge, 1989.

PREECE, J; ROGERS, Y; SHARP, H. Design de Interação: Além da Interação HomemComputador. Porto Alegre: Bookman, 2005.

SAFFER, D. Designing for Interaction: Creating Innovative Applications and Devices.

Berkeley, CA: New Riders Publishing, 2010.

SARACEVIC, T. Information Science. JASIS. 50(12), 1999. P. 1051-1068.

SIRIHAL, A. D. Mediação da informação e estudos de usuários: interrelações. InCID: Revista de Ciência da Informação e Documentação, América do Norte, 3, jun, 2012.

\title{
Agradecimento
}

O presente trabalho foi realizado com apoio da FAPEMIG.

\author{
EDITORA E GRÁFICA DA FURG \\ CÂMPUSCARREIROS \\ CEP 96203900 \\ editora@furg.br
}

